Pacific Journal of Mathematics

MANIFOLDS MODELLED ON THE DIRECT LIMIT OF LINES 


\title{
MANIFOLDS MODELLED ON THE DIRECT LIMIT OF LINES
}

\author{
RICHARD E. HEISEY
}

The main theorem of this paper is that topological manifolds modelled on $R^{\infty}=\lim _{\rightarrow} R^{n}$ are stable. Combined with previous work this theorem enables us to embed $R^{\infty}$-manifolds as open subsets of $R^{\infty}$, classify $R^{\infty}$-manifolds by homotopy type, and triangulate $R^{\infty}$-manifolds.

The results established here were announced in the [8].

1. Definitions and results. Let $R^{n}$ be the cartesian product of $n$ copies of $R$, where $R$ denotes the reals. Define $i_{n}: R^{n} \rightarrow R^{n+1}$ by $i_{n}\left(\left(x_{1}, \cdots, x_{n}\right)\right)=\left(x_{1}, \cdots, x_{n}, 0\right)$. Then $R^{\infty}=\lim \left\{R^{n} ; i_{n}\right\}$. We regard $R^{\infty}$ as the set $\left\{\left(x_{1}, x_{2}, x_{3}, \cdots,\right) \mid x_{i} \in R\right.$, all $i$, and $x_{i} \neq 0$ for at most finitely many $i\}$. We identify $R^{n}$ with $R^{n} \times\{(0,0, \cdots, 0)\} \subset R^{n+k}$, $k \geqq 1$, and with $R^{n} \times\{(0,0, \cdots)\} \subset R^{\infty}$. With this identification, a set $\mathcal{O} \subset R^{\infty}$ is open if and only if $\mathcal{O} \cap R^{n}$ is open in $R^{n}, n \geqq 1$. In the terminology of [14], for example, $R^{\infty}$ is thus the strict inductive limit of $\left\{R^{n}\right\}$. As such it is a locally convex [14, Prop. 1, p. 127], nonmetrizable [14, Prop. 5, p. 129] topological vector space having a natural simplicial structure.

A topological manifold modelled on $R^{\infty}$, or, more simply, an $R^{\infty}$-manifold, is a Hausdorff space in which each point has a neighborhood homeomorphic to an open subset of $R^{\infty}$. By way of example we note that countable direct limits of finite-dimensional manifold are often $R^{\infty}$-manifolds. Also by [9, Corollary 2], if $X$ is a locally finite polyhedron (more generally, a locally compact, locally finitedimensional ANR) then $X \times R^{\infty}$ is an $R^{\infty}$-manifold. Our main result is Theorem $\mathrm{S}$, below, which asserts that $R^{\infty}$-manifolds are stable with respect to multiplication by $R^{\infty}$. We remark that because $R^{\infty}$ is nonmetrizable and not a countable product (one can show that $R^{\infty}$ is not homeomorphic to $\left.R^{\infty} \times R^{\infty} \times R^{\infty} \times \cdots\right)$ many of the arguments used in establishing stability of Hilbert space and Hilbert cube manifolds as, for example, in [1] and [16] do not apply here. Our proof uses an inductive argument on finite-dimensional subsets.

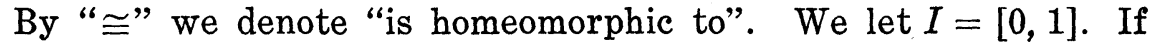
$\mathscr{U}$ is an open cover of the space $Y$, two maps $f, g: X Y$ are $\mathscr{W}$ close if for each $x \in X$ there is a $U \in \mathscr{C}$ such that $\{f(x), g(x)\} \subset U$. A map $f: X \rightarrow Y$ is a near homeomorphism if for each open cover $\mathscr{C}$ of $Y$ there is a homeomorphism $h: X \rightarrow Y$ such that $f$ and $h$ are $\mathscr{Q}$-close. 
For the remainder of this section let $M$ and $N$ denote paracompact, connected $R^{\infty}$-manifolds.

THEOREM $S$ (Stability). The projection $\operatorname{map} M \times R^{\infty} \rightarrow M$ is a near-homeomorphism. In particular $M \times R^{\infty} \cong M$.

The proof of the stability theorem is given in $\S 3$ of this paper.

In [7] it was shown that $M \times R^{\infty}$ embeds as an open subset of $R^{\infty}$. Combined with Theorem $\mathrm{S}$ this immediately implies the open embedding theorem for $R^{\infty}$-manifolds.

ThEOREM $\mathcal{O}$ (Open Embedding). There is an open embedding $f: M \rightarrow R^{\infty}$.

Using Theorem $\mathscr{O}$, regard $M$ as an open subset of $R^{\infty}$. Let $\mathscr{C}$ be an open cover of $M$ consisting of convex sets. By Theorem $\mathrm{S}$ there is a homeomorphism $h: M \times R^{\infty} \rightarrow M$ which is $\mathscr{C}$-close to the projection. Clearly, then, $H: M \times R^{\infty} \times I \rightarrow M$ defined by $H((m, x$, $t))=(1-t) h((m, x))+t m$ is a homotopy in $M$, and the following corollary results.

COROLLARY 1. There is a homeomorphism $h: M \times R^{\infty} \rightarrow M$ which is homotopic to the projection map.

Let $f: M \rightarrow N$ be a homotopy equivalence. By [7, Theorem II-9] $\left(f \times\right.$ id): $M \times R^{\infty} \rightarrow N \times R^{\infty}$ is homotopic to a homeomorphism $g$. Let $h_{M}: M \times R^{\infty} \rightarrow M$ and $h_{N}: N \times R^{\infty} \rightarrow N$ be homeomorphisms homotopic to the corresponding projection maps. Then $h_{N} g h_{M}^{-1}$ is a homeomorphism homotopic to $f$, and we have proven the following classification theorem.

TheOREM C (Classification by Homotopy Type). If $f: M \rightarrow N$ is a homotopy equivalence, then $f$ is homotopic to a homeomorphism $h: M \rightarrow N$.

Since $R^{\infty}$-manifolds have the homotopy type of ANR's [7, Theorem II-10], Theorem $\mathrm{C}$ has the following corollary.

Corollary 2. If $M$ and $N$ have the same weak homotopy type, then they are homeomorphic.

In [4] Dobrowolski obtains a special case of Corollary 2; namely, that $R^{\infty} \cong \lim S^{n}$, where $S^{n}$ is the $n$-sphere. He obtains this result by first showing that compact subsets of $\lim S^{n}$ are negligible. 
Using Theorems $\mathcal{O}$ and $\mathrm{C}$ we can now triangulate $M$. By Theorem $\mathcal{O}$ we may regard $M$ as an open subset of $R^{\infty}$. Since open subsets of $R^{\infty}$ are Lindelöf [7, Propositions III. 1 and III. 2] $M$ has the homotopy type of a countable, locally finite, simplicial complex $K$ [13, Theorem 1 and Proposition 2]. By [9, Corollary 2] $|K| \times R^{\infty}$ is an $R^{\infty}$-manifold, and clearly, $|K| \times R^{\infty}$ has the same homotopy type as $M$. By Theorem $\mathrm{C}, M \cong|K| \times R^{\infty}$. This establishes the triangulation theorem.

THEOREM T (Triangulation). $M \cong|K| \times R^{\infty}$, where $K$ is a countable, locally-finite simplicial complex.

We remark that Theorems $\mathrm{S}$ and $\mathrm{T}$ answer affirmatively two questions in the Appendix "Open problems of infinite-dimensional topology" in [3].

The author gratefully acknowledges several helpful conversations with Henryk Torunczyk and James West.

$\S 2$. Lemmas. Recall that we identify $R^{n}$ with $R^{n} \times\{0,0, \cdots, 0\} \subset$ $R^{n+k}$ and with $R^{n} \times\{0,0, \cdots\} \subset R^{\infty}$. If $A \subset R^{\infty}$, let $A^{n}=A \cap R^{n}$. Let $d_{n}$ be the metric induced on $R^{n}$ by the norm $\|x\|=\left(\sum x_{i}^{2}\right)^{1 / 2}$. If $\mathscr{Q}$ is an open cover of $Y$, a homotopy $H: X \times I \rightarrow Y$ is limited by $\mathscr{W}$ if for every $x \in X, H(\{x\} \times I) \subset U$, some $U \in \mathscr{U}$. We abbreviate "finite-dimensional" by f.d. and "piecewise linear" by p.l. If $A \subset X$, by $\bar{A}$ we denote the closure of $A$ in $X$.

LeMma 1. Let $A$ and $B$ be f.d. compact metric spaces with $A \subset B$. Let $f: B \rightarrow R^{n}$ be a continuous map such that $f / A$ is an embedding. Then if $m$ is sufficiently large, for every $\varepsilon>0$ there is an embedding $g_{\varepsilon}: B \rightarrow R^{m}$ such that $g_{\varepsilon} / A=f / A$ and $d_{m}\left(f, g_{\varepsilon}\right)<\varepsilon$.

Proof. We may assume that $2(\operatorname{dim} B)+1 \leqq n$ so that there is an embedding $\alpha: B \rightarrow R^{n}$. Let $\beta: R^{n} \rightarrow R^{n}$ be a continuous extension of $\alpha f^{-1}: f(A) \rightarrow R^{n}$. Define $h: B \rightarrow R^{n} \times R^{n}$ by $h(b)=(f(b), \alpha(b))$ and $T: R^{n} \times R^{n} \rightarrow R^{n} \times R^{n}$ by $T(x, y)=(x, y-\beta(x))$. Then $g=T h$ : $B \rightarrow R^{n} \times R^{n}$ is an embedding extending $f / A$. Choose $r>0$ such that $g(B) \subset R^{n} \times\left\{z \in R^{n} \mid\|z\| \leqq r\right\}$. If $e(x, y)=(x,(\varepsilon / r) y)$, then $g_{\varepsilon}=$ $e g$ is the desired embedding.

LemMa 2. Let $X$ be a f.d. locally compact metric space and $A$ and $B$ closed sets in $X$ such that $X=A \cup B$ and $B$ is compact. Let $U$ be an open subset of $R^{\infty}, \mathscr{C}$ an open cover of $U$. Let $f: X \rightarrow U$ be a continuous map such that $f / A$ is a closed embedding. Then there is an embedding $g: X \rightarrow U$ such that $g / A=f / A$ and such that 
$H: X \times I \rightarrow U$ defined by $H(x, t)=(1-t) f(x)+t g(x)$ is limited by $\mathscr{U}$.

Proof. If $C$ is a compact subset of $U$, then $f^{-1}(C)$ is contained in the compact set $(f / A)^{-1}(C) \cup B$. Hence $f$ is proper. Thus, we can choose a relatively compact neighborhood $V$ of the compact set $f^{-1}(f(B))$ in locally compact $X$.

Let $n$ be such that $f(\bar{V}) \subset U \cap R^{n}$. By Lemma 1 there is an $m>n$ and an embedding $g_{\varepsilon}: \bar{V} \rightarrow R^{m}$ with $g_{\varepsilon}(x)=f(x)$ for $x \in A \cap \bar{V}$ and $d_{m}\left(g_{\varepsilon}, f / \bar{V}\right)<\varepsilon$, where $\varepsilon>0$ is chosen smaller than $d_{m}(f(B)$, $\left.f(A \backslash V) \cap R^{m}\right)$ and such that the $\varepsilon$-neighborhood in $R^{m}$ of any point of $f(\bar{V})$ is contained in a member of $\left\{W \cap R^{m} \mid W \in \mathscr{C}\right\}$. Define $g: X \rightarrow U$ by $g(x)=f(x)$ for $x \in A$ and $g(x)=g_{\varepsilon}(x)$ for $x \in \bar{V}$. Thus, $g$ is one-to-one. Moreover, $g$ is proper, for the same reason for which $f$ is. It follows that $g$ is the desired embedding.

Lemma 3. Let $A$ and $B$ be f.d. compact metric spaces with $A \subset B$. Let $M$ be a paracompact space such that $M=U_{1} \cup U_{2}$, where $U_{i}, i=1,2$, is an open subset of $M$ homeomorphic to an open subset of $R^{\infty}$. Let $f: B \rightarrow M$ be a continuous map such that $f / A$ is an embedding. Then there is an embedding $f^{\prime}: B \rightarrow M$ such that $f^{\prime} / A=f / A$.

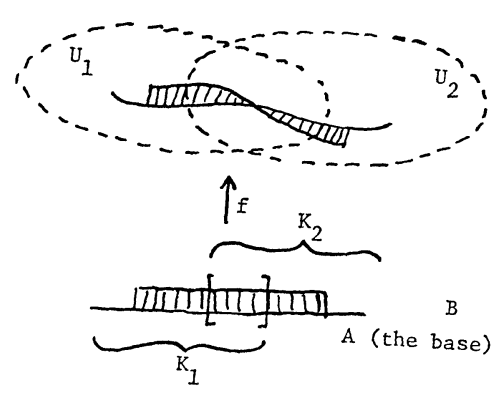

Let $\left\{K_{1}, K_{2}\right\}$ be a cover of $B$ by compact sets such that $K_{1} \subset$ $f^{-1}\left(U_{i}\right), i=1,2$. By Lemma 2 there is an embedding $g_{1}: K_{1} \cup[A \cap$ $\left.f^{-1}\left(U_{1}\right)\right] \rightarrow U_{1}$ such that $g_{1}(x)=f(x)$ for $x \in A \cap f^{-1}\left(U_{1}\right)$ and such that $f / K_{1} \cup\left[A \cap f^{-1}\left(U_{1}\right)\right]$ is homotopic to $g_{1}$ by a homotopy $H$ fixed on $A \cap f^{-1}\left(U_{1}\right)$ and limited by $\left\{U_{1} \cap U_{2}, M \backslash f\left(K_{1} \cap K_{2}\right)\right\}$. Note that $H\left[\left(K_{1} \cap K_{2}\right) \times I\right] \subset U_{1} \cap U_{2}$. Define $H^{\prime}:\left[\left(K_{1} \cap K_{2}\right) \cup\left(A \cap K_{2}\right)\right] \rightarrow U_{2}$ by $H^{\prime}(x, t)=H(x, t)$ for $x \in K_{1} \cap K_{2}, t \in I$, and $H^{\prime}(x, t)=f(x), x \in A \cap K_{2}$, $t \in I$.

By Dugundij's theorem [5, p. 188], $R^{\infty}$, and, hence, [10, p. 42], $U_{2}$ is an absolute neighborhood extensor for the class of metrizable spaces. It follows, as in the proof of [10, Theorem 2.2, p. 117], that $U_{2}$ has the homotopy extension property with respect to metric 
spaces. Since $H_{0}^{\prime}=f / A \cap K_{2}$ extends by $f$ to all of $K_{2}, H^{\prime}$ has an extension $\bar{H}: K_{2} \times I \rightarrow U_{2}$. Define $g: B \rightarrow U_{1} \cup U_{2}$ by $g / K_{1}=g_{1} / K_{1}$ and $g / K_{2}=\bar{H}_{1}$. Then $g$ extends $f / A$.

By Lemma 2 there is an embedding $g_{2}: g^{-1}\left(U_{2}\right) \rightarrow U_{2}$ such that $g_{2}(x)=g(x)$ for $x \in g^{-1}\left(U_{2}\right) \cap\left(A \cup K_{1}\right)$. Define $f^{\prime}: B \rightarrow U_{1} \cup U_{2}$ by $f^{\prime}(x)=g_{2}(x)$ if $x \in g^{-1}\left(U_{2}\right)$ and $f^{\prime}(x)=g(x)$ otherwise. Then $f^{\prime} / K_{1}=$ $g_{1} / K_{1}$ and $f^{\prime} / K_{2}=g_{2} / K_{2}$ so that $f^{\prime}$ is continuous. If $f^{\prime}(x)=f^{\prime}(y)$, then either both $x$ and $y$ or neither $x$ nor $y$ is in $\left(f^{\prime}\right)^{-1}\left(U_{2}\right)=g^{-1}\left(U_{2}\right)$. In the first case $x=y$ since $g_{2}$ is one-to-one. In the latter case $x=y$ since $g / K_{1}$ is one-to-one. Clearly $f^{\prime} / A=f / A$. Thus, $f^{\prime}$ is the desired embedding.

The last lemma is probably known. We include a proof for completeness.

Lemma 4. Let $X$ be a finite polyhedron and $M$ a compact p.l. manifold with boundary. If $f, g: X \rightarrow$ Int $M$ are homotopic topological embeddings, then for sufficiently large $k$ there is an ambient isotopy $H$ on $M \times[-1,1]^{k}$ such that $H_{1}(f, 0)=(g, 0): X \rightarrow M \times[-1,1]^{k}$.

Proof. Let $H: X \times I \rightarrow$ Int $M$ be a homotopy with $H_{0}=f$ and $H_{1}=g$. Define $\bar{H}: X \times I \rightarrow \operatorname{Int}\left(M \times[-1,1]^{k}\right)$ by $\bar{H}(x, t)=(H(x, t)$, $t / 2,0,0, \cdots, 0)$. Then $\bar{H}_{0}=(f, 0)$ and $\bar{H}_{1}=(g, 1 / 2,0, \cdots, 0)$. Clearly it is sufficient to show that $(f, 0)$ and $\bar{H}_{1}$ are ambient isotopic.

Since $\bar{H} / X \times\{0,1\}$ is an embedding, Theorem 1 of [2] implies that for sufficiently large $k, \bar{H} / X \times\{0,1\}$ is $\varepsilon$-tame in $\operatorname{Int}(M \times$ $\left.[-1,1]^{k}\right)$. Thus, there is an ambient isotopy $K_{t}: M \times[-1,1]^{k} \rightarrow M \times$ $[-1,1]^{k}$ such that $K_{t}(\bar{H}(X \times I)) \subset \operatorname{Int}\left(M \times[-1,1]^{k}\right), t \in I$, and such that $K_{1} \bar{H} / X \times\{0,1\}$ is a p.l. embedding. Using general position $[15,5.4$, p. 61] there is, for sufficiently large $k$, a p.l. embedding $h: X \times I \rightarrow \operatorname{Int}\left(M \times[-1,1]^{k}\right)$ such that $h / X \times\{0,1\}=K_{1} \bar{H} / X \times\{0,1\}$. By [11, Theorem 1.1, p. 426] there is an ambient isotopy $E_{t}: M \times$ $[-1,1]^{k} \rightarrow M \times[-1,1]^{k}$ such that $E_{1} h_{0}=h_{1}$. Then $K_{t}^{-1} E_{t} K_{t}$ is an ambient isotopy on $M \times[-1,1]^{k}$ with $K_{1}^{-1} E_{1} K_{1}(f, 0)=K_{1}^{-1} E_{1} K_{1} \bar{H}_{0}=$ $K_{1}^{-1} E_{1} h_{0}=K_{1}^{-1} h_{1}=\bar{H}_{1}$, as required.

3. Proof of Theorem S. We first prove the following weaker version of the stability theorem.

THEOREM S'. Let $M$ be a paracompact $R^{\infty}$-manifold such that $M=U \cup V$, where $U$ and $V$ are homeomorphic to open subsets of $R^{\infty}$. Then there is a homeomorphism $M \rightarrow M \times R^{\infty}$.

Proof. We first show that $M$ can be suitably expressed as the 
direct limit of topological manifolds. Let $\gamma: U \rightarrow U^{\prime}$ and $\delta: V \rightarrow V^{\prime}$ be homeomorphisms onto open subsets of $R^{\infty}$. Then $U^{\prime}=\lim C_{n}^{\prime}$ where $C_{n}^{\prime}$ is a compact metric subspace of $U^{\prime} \cap R^{n}$ and where $\vec{C}_{n}^{\prime} \subset$ Int $_{n+1} C_{n+1}$. Express $V^{\prime}=\lim D_{n}^{\prime}$ similarly. Let $C_{n}=\delta^{-1}\left(C_{n}^{\prime}\right)$ and $D_{n}=\delta^{-1}\left(D_{n}^{\prime}\right)$.

Fix $n \geqq 1$. Since $C_{n} \cup D_{n}$ is a compact f.d. metric space, there is an embedding $\alpha: C_{n} \cup D_{n} \rightarrow R^{k}$, some $k$. Since $M$ is an absolute neighborhood extensor for metric spaces ([5, p. 188] and [10, p. 45]), $\alpha^{-1}: \alpha\left(C_{n} \cup D_{n}\right) \rightarrow M$ has a continuous extension $\beta$ to a compact p.l. submanifold $N$ of $R^{k}$ containing $\alpha\left(C_{n} \cup D_{n}\right)$. Let $\pi: N \times I \rightarrow N$ be the projection. Then $\beta \pi: N \times I \rightarrow M$ is an embedding on $\alpha\left(C_{n} \cup\right.$ $\left.D_{n}\right) \times\{0\}$. By Lemma 4 there is an embedding $\beta^{\prime}: N \times I \rightarrow M$ such that $\beta^{\prime}\left(\alpha\left(C_{n} \cup D_{n}\right) \times\{0\}\right)=\beta \pi\left(\alpha\left(C_{n} \cup D_{n}\right) \times\{0\}\right)=C_{n} \cup D_{n}$. Let $X=$ $\partial(N \times I)$, a closed p.l. manifold. Let $X_{n}=\beta^{\prime}(X)$. Note that $X_{n} \supset$ $C_{n} \cup D_{n}$ and, since $M=\lim \left(C_{n} \cup D_{n}\right), M=\lim X_{n}$.

Let $A \subset M$ be a compact subspace. Choose an open cover $\left\{Y_{1}\right.$, $\left.Y_{2}\right\}$ of $M$ such that $\bar{Y}_{1} \subset U$ and $\bar{Y}_{2} \subset V$. Then $A=\left(A \cap \bar{Y}_{1}\right) \cup(A \cap$ $\left.\bar{Y}_{2}\right)$. The compactness of $A \cap \bar{Y}_{1}$ and $A \cap \bar{Y}_{2}$ implies that for some $n, \gamma\left(A \cap \bar{Y}_{1}\right) \subset C_{n}^{\prime}$ and $\delta\left(A \cap \bar{Y}_{2}\right) \subset D_{n}^{\prime}$ so that $A \subset C_{n} \cup D_{n}$. Thus, every compact subspace of $M$ is contained in some $X_{n}$.

Now, let $B_{n}=[-n, n]^{n}, n \geqq 1$. Then $R^{\infty}=\lim B_{n}$. Define $j_{n, k}^{\prime}$ : $X_{n} \times B_{k} \rightarrow M$ by $j_{n, k}^{\prime}(x, t)=x$. By Lemma 4 there is an embedding $j_{n, k}: X_{n} \times B_{k} \rightarrow M$ such that $j_{n, k}(x, 0)=x$ for each $x \in X_{n}$.

Let $j_{1}=j_{1,1}$. Choose $n_{2}>1$ such that $j_{1}\left(X_{1} \times B_{1}\right) \subset X_{n_{2}}$. Consider

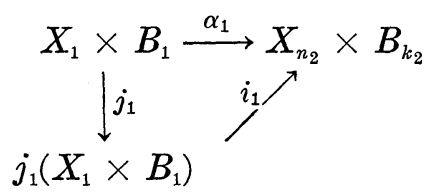

where $k_{2}>1$ is yet to be chosen, $i_{1}(y)=(y, 0)$ and $\alpha_{1}(x, t)=(x,(t, 0))$. Since $B$ is contractible $i_{1} j_{1} \sim \alpha_{1}$ (" $\sim$ " denotes "is homotopic to") with the homotopy taking place in Int $\left(X_{n_{2}} \times B_{k_{2}}\right)$. Choose $k_{2}$ so large that, by Lemma 4 , there is an ambient isotopy $F_{2}$ on $X_{n_{2}} \times$ $B_{k_{2}}$ such that $\left(F_{2}\right)_{1} \alpha_{1}=i_{1} j_{1}$. Let $j_{2}=j_{n_{2}, k_{2}}: X_{n_{2}} \times B_{k_{2}} \rightarrow j_{2}\left(X_{n_{2}} \times B_{k_{2}}\right)$. Let $h_{1}=j_{1}$ and $h_{2}=j_{2}\left(F_{2}\right)_{1}$. Consider

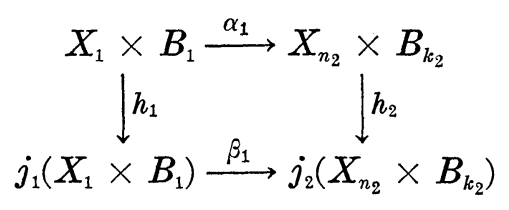

where $\beta_{1}(y)=y$. Since $h_{2} \alpha_{1}=j_{2}\left(F_{2}\right)_{1} \alpha_{1}=j_{2} i_{1} j_{1}=\beta_{1} j_{1}$, the square commutes. Also, $((y, t), s) \rightarrow j_{2} F_{2}((y, t), s)$ defines a homotopy from 
$j_{2}$ to $h_{2}$.

Choose $n_{3}>n_{2}$ such that $j_{2}\left(X_{n_{2}} \times B_{k_{2}}\right) \subset X_{n_{3}}$. Consider

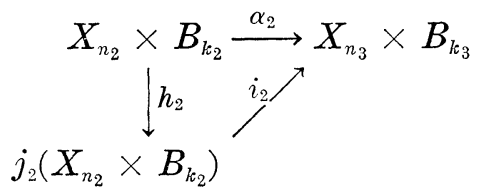

where $k_{3}>k_{2}$ is yet to be chosen, $\alpha_{2}(x, t)=(x,(t, 0))$ and $i_{2}(y)=(y, 0)$. Since $j_{2} \sim h_{2}$, we obtain homotopies $i_{2} h_{2} \sim i_{2} j_{2} \sim \alpha_{2}$ taking place in Int $\left(X_{n_{2}} \times B_{k_{2}}\right)$. By Lemma 4 we may choose $k_{3}$ so large that there is an ambient isotopy $F_{3}$ on $X_{n_{3}} \times B_{k_{3}}$ such that $\left(F_{3}\right)_{1} \alpha_{2}=i_{2} h_{2}$. Let $j_{3}=j_{n_{3}, k_{3}}$ and $h_{3}=j_{3}\left(F_{3}\right)_{1}$.

Continuing, by induction we obtain for every $r \geqq 1$ a commutative diagram

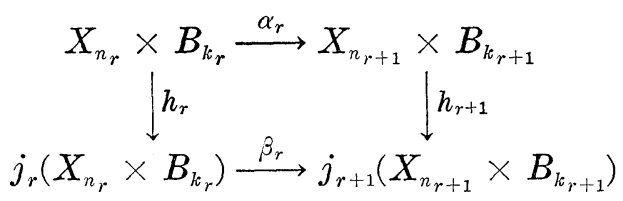

where $\alpha_{r}(x, t)=(x,(t, 0)), \beta_{r}(y)=y$ and $h_{r}$ is a homeomorphism. Let $D=\lim \left\{X_{n_{r}} \times B_{k_{r}} ; \alpha_{r}\right\}$ and $E=\lim \left\{j_{r}\left(X_{n_{r}} \times B_{k_{r}}\right) ; \beta_{r}\right\}$. The $h_{r}$ 's induce a homeomorphism $h: D \rightarrow \vec{E}$. As sets clearly $D \equiv M \times R^{\infty}$ and $E \equiv M$. Since $j_{r}\left(X_{n_{r}} \times B_{n_{r}}\right) \supset X_{n_{r}}$ and $M=\lim X_{n}$ it follows immediately that $E \cong M$. Also, $M \times R^{\infty}$ is homeomorphic to an open subset of $R^{\infty}$ [7, Corollary II-7] and is therefore the direct limit of its compact subsets. If $C \subset M \times R^{\infty}$ is compact, then $C \subset \pi_{1}(C) \times$ $\pi_{2}(C) \subset X_{n_{r}} \times B_{k_{r}}$ some $r$. (Here $\pi_{1}: M \times R^{\infty} \rightarrow M$ and $\pi_{2}: M \times R^{\infty} \rightarrow$ $R^{\infty}$ are the projections.) It follows that $D \cong M \times R^{\infty}$. Thus, $M \cong$ $M \times R^{\infty}$, and Theorem $\mathrm{S}^{\prime}$ is proved.

Theorem $\mathrm{S}$ now follows quickly. Let $M$ be a paracompact, connected $R^{\infty}$-manifold. As shown in [7, Proposition III. 1] every subset of $M$ is paracompact. Say that a paracompact space $Z$ has property $P$ if for every open subset $U$ of $Z$ there is an open embedding $U \rightarrow R^{\infty}$. Then $M$ has property $P$ locally. Let $X=U U$ $V \subset M$ where $U$ and $V$ are open in $M$ having property $P$. By Theorem $\mathrm{S}^{\prime} X \cong X \times R^{\infty}$. By [7, Corollary II.7] $X$ has property $P$. Let $Y=\mathrm{U}_{i} Y_{i}$ where $Y_{i}$ is open in $M$ and has property $P$, and where $\left\{Y_{2}\right\}$ is discrete. Since $M$ is Lindelöf [7, Proposition III. 1], $\left\{Y_{i}\right\}$ is at most countable, indexed, say, by a subset of the integers. Let $f_{i}: Y_{i} \rightarrow R^{\infty}$ be an open embedding. Let $\rho_{i}: R^{\infty} \rightarrow[(i-1 / 3, i+$ $1 / 3) \times R \times R \times \cdots] \cap R^{\infty}$ be a homeomorphism. Then $f: Y \rightarrow R^{\infty}$ defined by $f / Y_{i}=\rho_{i} \circ f_{i}$ is an open embedding, showing that $Y$ has 
property $P$. By a theorem of Michael [12, Theorem 3.6] $M$ has property $P$. That is, there is an open embedding $M \rightarrow R^{\infty}$. By [6, Theorem 1] the projection $\pi: M \times R^{\infty} \rightarrow M$ is then a near homeomorphism. This completes the proof of Theorem S.

\section{REFERENCES}

1. R. D. Anderson and R. Schori, Factors of infinite-dimensional manifolds, Trans. Amer. Math. Soc. 142 (1969), 315-330.

2. J. L. Bryant, Taming polyhedra in the trivial range, Michigan Math. J., 13 (1966), 377-384.

3. T. A. Chapman, Lectures on Hilbert cube manifolds, CBMS Number 28, Amer. Math. Soc., 1976.

4. T. Dobrowolski, Extensions of Bessaga's negligibility technique to certain infinitedimensional groups, Bull. Acad. Polon., Ser. Sci. Math. Astronom. Phys., 26 (1978).

5. J. Dugundji, Topology, Allyn and Bacon, Boston, 1966.

6. R. E. Heisey, Factoring open subsets of $R^{\infty}$ with control, to appear in Topology Proceedings.

7. - Manifolds modelled on $R^{\infty}$ or bounded weak-* topologies, Trans. Amer. Math. Soc., 206 (1975), 295-312.

8. - Stability, classification, open embeddings, and triangulation of $R^{\infty}$-manifolds, submitted to the Proceedings of the Conference on Geometric Topology, Warsaw, 1978.

9. D. W. Henderson, A simplicial complex whose product with any ANR is a simplicial complex, General Topology and Appl., 3 (1973), 81-83.

10. S. T. Hu, Theory of Retracts, Wayne State Univ. Press, Detroit, 1965.

11. J. F. P. Hudson, Concordance, isotopy, and diffeotopy, Annals of Math., 2nd Series, 91 (1970), 425-448.

12. E. A. Michael, Local properties of topological spaces, Duke Math. J., 21 (1954), 163-172.

13. J. Milnor, On spaces having the homotopy type of a $C W$-complex, Trans. Amer. Math. Soc., 90 (1959), 272-280.

14. A. P. Robertson, and W. Robertson, Topological Vector Spaces, second edition, Cambridge Univ. Press, 1973.

15. C. P. Rourke and B. J. Sanderson, Intro. to Piecewise Linear Topology, Ergeberisse Math. Grenz. Band 69, Springer-Verlag, N. Y., 1972.

16. Schori, Topological stability for infinite-dimensional manifolds, Compositio Math., 23 (1971), 87-100.

Received June 15, 1979 and in revised form February 9, 1980.

VANDERBILT UNIVERSITY

NASHVILLE, TN 37235 


\section{PACIFIC JOURNAL OF MATHEMATICS}

\section{EDITORS}

DONALD BABBITT (Managing Editor)

University of California

Los Angeles, California 90024

HUGO ROSSI

University of Utah

Salt Lake City, UT 84112

C. C. MOORE and ARTHur AGUS

University of California

Berkeley, CA 94720
J. DUGUNDJI

Department of Mathematics

University of Southern California

Los Angeles, California 90007

R. FINN and J. MILGRAM

Stanford University

Stanford, California 94305

\section{ASSOCIATE EDITORS}
R. Arnes
E. F. BECKENBACH
B. H. NeumanN
F. WOLF
K. YösHIDA

\section{SUPPORTING INSTITUTIONS}

UNIVERSITY OF ARIZONA

UNIVERSITY OF BRITISH COLUMBIA

CALIFORNIA INSTITUTE OF TECHNOLOGY

UNIVERSITY OF CALIFORNIA

MONTANA STATE UNIVERSITY

UNIVERSITY OF NEVADA, RENO

NEW MEXICO STATE UNIVERSITY

OREGON STATE UNIVERSITY
UNIVERSITY OF OREGON

UNIVERSITY OF SOUTHERN CALIFORNIA

STANFORD UNIVERSITY

UNIVERSITY OF HAWAII

UNIVERSITY OF TOKYO

UNIVERSITY OF UTAH

WASHINGTON STATE UNIVERSITY

UNIVERSITY OF WASHINGTON 


\section{Pacific Journal of Mathematics}

Vol. 102, No. $1 \quad$ January, 1982

S. Agou, Degré minimum des polynômes $f\left(\sum_{i=0}^{m} a_{i} X^{p^{r i}}\right)$ sur les corps finis

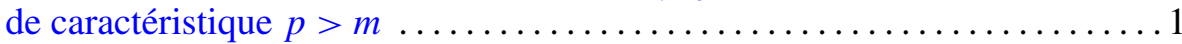

Chi Cheng Chen, On the image of the generalized Gauss map of a complete

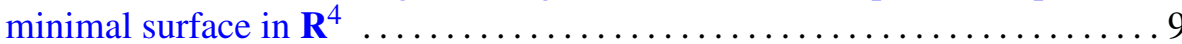

Thomas Curtis Craven and George Leslie Csordas, On the number of real

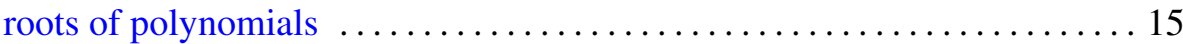

Allan L. Edelson and Kurt Kreith, Nonlinear relationships between oscillation and asymptotic behavior ....................... 29

B. Felzenszwalb and Antonio Giambruno, A commutativity theorem for

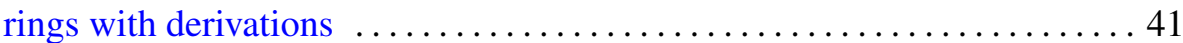

Richard Elam Heisey, Manifolds modelled on the direct limit of lines . . . . . 47

Steve J. Kaplan, Twisting to algebraically slice knots $\ldots \ldots \ldots \ldots \ldots \ldots 5$

Jeffrey C. Lagarias, Best simultaneous Diophantine approximations. II.

Behavior of consecutive best approximations $\ldots \ldots \ldots \ldots \ldots \ldots \ldots 61$

Masahiko Miyamoto, An affirmative answer to Glauberman's conjecture . . . 889

Thomas Bourque Muenzenberger, Raymond Earl Smithson and L. E.

Ward, Characterizations of arboroids and dendritic spaces ........... 107

William Leslie Pardon, The exact sequence of a localization for Witt

groups. II. Numerical invariants of odd-dimensional surgery

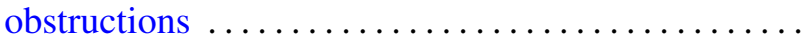

Bruce Eli Sagan, Bijective proofs of certain vector partition identities

Kichi-Suke Saito, Automorphisms and nonselfadjoint crossed products ...

John Joseph Sarraille, Module finiteness of low-dimensional PI rings ...

Gary Roy Spoar, Differentiable curves of cyclic order four . .

William Charles Waterhouse, Automorphisms of quotients of $\Pi \mathrm{GL}\left(n_{i}\right)$

Leslie Wilson, Mapgerms infinitely determined with respect to right-left equivalence

Rahman Mahmoud Younis, Interpolation in strongly logmodular

algebras 